2. Second Term Time Series Analysis; Scaling and Dimensional Analysis; Mathematical Models: Rational Choice; Introduction to Statistics and Data Analysis II; Categorical Data Analysis; „LISREL“ Models: General Structural Equations; Complex System Models in the Social Sciences; Simultaneous Equation Models; Regression Analysis.

Das Programm ist umfangreicher als hier dargestellt. Das ICPSR veröffentlicht das gesamte Ausbildungsangebot auf seinen Internetseiten. Wenngleich sich unsere Darlegung auf das Summer Program vom letzten Jahr bezieht, kann im Allgemeinen davon ausgegangen werden, dass sich an der Grundstruktur des Angebotes in diesem Jahr wenig ändert. Die Programmübersicht für das Jahr 2006 wird in der Regel im März verfügbar gemacht. Die Anmeldung erfolgt bis Ende April. Über Möglichkeiten der Finanzierung eines Aufenthaltes in Ann Arbor sollten sich Interessenten frühzeitig informieren.

Ansprechpartnerin im Zentralarchiv ist Maria Rohlinger, Tel.: 0221/47694-45,

E-Mail: rohlinger@za.uni-koeln.de.

Weitere Informationen zum Summer Program findet man auch im Internet unter der http:// www.icpsr.umich.edu (unter „Education and Instruction").

Forschung und Analysemöglichkeiten mit der Einkommens- und Verbrauchsstichprobe (EVS); 1. Nutzerkonferenz

19./20. Oktober 2006, Mannheim

Die Einkommens- und Verbrauchsstichprobe (EVS) wird vom Statistischen Bundesamt seit 1962 in 5-Jahres-Abständen durchgeführt, zuletzt in 2003. Die EVS ist die einzige Erhebung in Deutschland, die die Ausstattung privater Haushalte mit Gebrauchsgütern, ihre Einkommen und Vermögen als auch ihren privaten Konsum in detaillierter Weise anhand verschiedener Erhebungsteile und -unterlagen ermittelt.

Die Daten aller Jahrgänge der EVS werden 2006 erstmals vollständig als Mikrodaten für die Forschung in den empirischen Sozial- und Wirtschaftswissenschaften verfügbar sein.

Die Ziele der ersten EVS-Nutzerkonferenz sind: Information über das Analysepotential der EVS-Mikrodaten, Diskussion über neuere Forschungsergebnisse, die auf der Basis dieser Daten gewonnen wurden, Erfahrungsaustausch zwischen Datennutzern und Datenproduzenten.

Die Konferenz wendet sich an Forscher, die bereits mit den Mikrodaten der EVS arbeiten. Sie ist eine gemeinsame Veranstaltung von ZUMA und dem Statistischen Bundesamt.

Konferenzanmeldung: ZUMA-Tagungssekretariat; Postfach 122155, D-68072 Mannheim, Tel.: 0621/1246-221, Fax: 0621/1246-100; E-Mail: workshop@zuma-mannheim.de; Ansprechpartner bei ZUMA: Dr. Georg Papastefanou, E-Mail: papastefanou@zuma-mannheim.de

\section{CALL FOR PAPERS}

"Schönheit, Schutz und Pflege.

Zur Professionalisierung körperbezogener Dienstleistungen"

Tagung der DGS-Arbeitsgruppe Professionssoziologie Johann Wolfgang Goethe Universität Frankfurt a.M., 4.15. Mai 2006

Professionalisierungsbestrebungen dokumentieren in der Regel den Anspruch auf gesellschaftliche Anerkennung eines Kompetenzprofils sowie entsprechende Erhöhung der Gratifikation. Diesseits der historisch längst überschrittenen Sattelzeit erfolgreich durchgesetzter Zuständigkeitsmonopole, mit denen sich die Rechtspflege, Therapie, Kunst und Wissenschaft in einer Vielzahl von beruflichen Spezialisierungen und in jeweils unterschiedlichen organisatorischen Kontexten haben etablieren können, entfalten sich - nach neu entstandener oder behaupteter Bedürfnislage - im Horizont der klassischen Professionen Kompetenzsegmente, die Zuständigkeit, Gratifikation und Anerkennung reklamieren und somit ihrerseits in Konkurrenz zum professionellen Establishment sowie zu Nachbarkompetenzen geraten.

Die Tagung setzt sich zum Ziel, empirische Befunde zur Ausweitung von Professionalitätsansprüchen zu diskutieren und diese im Hinblick auf die theoretische Diskussion zur Professionalität von Dienstleistungen zu beziehen. Der Fokus der Aufmerksamkeit von theoretischen oder empirischen Beiträgen liegt auf der Analyse von körperbezogenen Dienstleistungen, im weitesten Sinn die Schönheit, den Schutz und die Pflege der Person betreffend.

Referatsangebote sollten bis spätestens 31 . März 2006 bei Tilman Allert (allert@soz.unifrankfurt.de, Tel. 069/798-22475, Fachbereich Gesellschaftswissenschaften, Robert-Mayer-Str. 5, 60054 Frankfurt a.M.) eingehen. 